\title{
Screening of Maternal Toxoplasmosis in Pregnancy: Laboratory Diagnostics from the Perspective of Public Health Requirements
}

\section{Sagel $\mathbf{U}^{1,2 *}$ and Krämer $\mathbf{A}^{1}$}

${ }^{1}$ Department of Public Health Medicine, School of Public Health, University of Bielefeld, P.O. Box 1001 31, D-33501 Bielefeld, Germany

${ }^{2}$ Institute of Hygiene and Microbiology, Lower Austria State Hospital of St. Pölten-Lilienfeld, Propst-Führer-Str. 4, A-3100 St. Pölten, Austria

\begin{abstract}
Serological screening for maternal Toxoplasma infections in pregnancy has been questioned recently. We analyze some diagnostic difficulties for routine laboratories, poor public health guidance of existing screening programs, and their mutual worsening impact on the efficacy of the programs and on toxoplasmosis research. False positive screening tests may be more likely than true maternal Toxoplasma infections and diagnosis often depends on confirmatory testing in experienced reference laboratories. Apart from clear seroconversions, any marker to assign the time point of infection to the ongoing pregnancy (IgM, IgG avidity, etc.) suffers from important limitations. With poor screening compliance, many screening alerts come from first serum samples in pregnancy that are cumbersome to test, while seroconversions are seldom observed due to missing follow-up samples in late pregnancy. From a public health perspective, inadequate epidemiological assessment and research, insufficient quality control for compliance and little consideration of diagnostic peculiarities for the design of more effective preventive programs has resulted in poor performance. These shortcomings have contributed to the present doubts about preventive Toxoplasma screening in pregnancy. We recommend that a team of public health decision makers, epidemiologists and experts from toxoplasmosis reference laboratories reevaluates the existing activities in a given country to build up a well-designed preventive program that avoids these drawbacks.
\end{abstract}

Keywords: Screening; toxoplasmosis; pregnancy; Toxoplasma infections

\section{Introduction}

For more than three decades, mother-child care programs have included screening for Toxoplasma infections in pregnancy by means of laboratory tests from blood samples in some countries (esp. Austria and France) [1-3]. Recently, despite much international research, the efficiency of this approach in preventing toxoplasmosis in the fetus has been questioned, more on the ground of inconclusive data than on convincing study results [4-8].

We will discuss here how poor public health guidance on laboratory screening has contributed to this unfortunate development and how to improve the existing programs, e.g. in Austria. Emphasis is placed on particular aspects of the routine laboratory in this process, while reference testing and management of prenatal toxoplasmosis is dealt with only briefly. Neonatal screening for congenital toxoplasmosis will not be discussed here, as recent research suggests that, aside from general doubts about treatment efficacy, an early treatment in pregnancy is the most promising approach $[4,9]$.

\section{Toxoplasmosis in Pregnancy}

Toxoplasmosis is a protozoan disease caused by Toxoplasma gondii. Cats are the definitive hosts, shedding oocysts that become infective a few days later. Many animals may be infected to become intermediate hosts, carrying infective cysts in various organs including muscles. Closing the cycle, cats typically become infected when eating rodents that contain cysts. Humans acquire infection from ripe oocysts or eating meat from infected animals (e.g. pork). In immunocompetent persons and pregnant women, infection rarely produces symptoms (like lymphadenitis). Within a few weeks, infection is limited to a few cysts followed by life-long specific immunity unless hampered by severe immunodeficiency (e.g. cancer, AIDS).

Women who are infected for the first time in their life by the parasite ("primoinfection") lack protective immunity. In these cases,
Toxoplasma gondii may cross the placental border and infect the fetus. Depending on the gestational week, infection may cause, to various probabilities, different afflictions of the central nervous system, such as hydrocephalus, intracranial calcifications and retinochorioiditis leading to life-long disabilities in children. Often, congenital toxoplasmosis is asymptomatic at birth and sequels may appear later in life [10] leading to underreporting if the outcome is only studied at delivery.

Havelaar et al. [11] compared the public health impact of congenital toxoplasmosis to salmonellosis as another important zoonotic disease. Although the latter is much more frequent in the general population of the Netherlands, the burden of disease of both conditions has been calculated to be similar. This is due to severe and long-lasting sequels in patients suffering from congenital toxoplasmosis [11].

In women who have already had Toxoplasma infection before pregnancy, immunity usually prevents access of the pathogen to the fetus, and it is regarded as protective. Therefore, preventive efforts focus on seronegative women who can be clearly distinguished from "immune" women, who will remain seropositive throughout their child-bearing lives.

Folic acid antagonists have proved to be an effective treatment in primoinfections and recurrences of immunocompromised patients.

*Corresponding author: Dr. Ulrich Sagel, Institute for Hygiene and Microbiology, Regional Hospital St. Poelten-Lilienfeld, Propst-Fuehrer-Str. 4, A-310S0 St. Poelten Austria, Tel: ++43-2742-300-13223; E-mail: ulrich.sagel@stpoelten.lknoe.at

Received October 18, 2012; Accepted January 25, 2013; Published February 10,2013

Citation: Sagel U, Krämer A (2013) Screening of Maternal Toxoplasmosis in Pregnancy: Laboratory Diagnostics from the Perspective of Public Health Requirements. J Bacteriol Parasitol S5-003. doi:10.4172/2155-9597.S5-003

Copyright: (c) 2013 Sagel U, et al. This is an open-access article distributed under the terms of the Creative Commons Attribution License, which permits unrestricted use, distribution, and reproduction in any medium, provided the original author and source are credited. 
Therefore, and from personal experience, toxoplasmosis experts believed for some decades that early treatment of maternal infections in pregnancy prevented or cured transmission and adverse sequels in the fetus. But early treatment requires serological screening as the vast majority of maternal infections go clinically unperceived [12-14].

\section{Prevention of toxoplasmosis by mother-child care programs}

In about 1975, Austria was the first country, soon to be followed by France, to introduce a free population-wide screening for primoinfections into its general mother-child care program. A few other countries joined this approach later (e.g., some regions of Italy and Slovenia). Denmark restricted its program to newborn screening of specific IgM antibodies in cord blood, believing that late treatment would still be beneficial for the offspring [15]. In many European countries, there is some kind of "wild screening", i.e., screening is neither recommended on a national level nor free for all women, but due to the advocacy of scientific associations or even official institutions, it is widely practiced [2].

Recently, large European multicenter studies have questioned the effectiveness of preventive treatment of maternal infections in pregnancy [4-7]. Due to this news, many public health authorities and health care payment providers have withdrawn their support for screening (e.g., Denmark stopped its neonatal screening in 2007 [1] and Switzerland even voted against voluntary screening except for a surveillance project in the Basel region in 2008 [16]). Nonetheless, many limitations and biases of these studies have been discussed [8] and many toxoplasmosis experts propose important arguments in favor of the treatment concept [9,13,17-19]. At present, further high quality studies are urgently needed to clarify the issue of treatment effectiveness [20].

\section{Laboratory tests for screening and confirmation of maternal Toxoplasma infections}

Screening tests and algorithm: Screening for maternal Toxoplasma primoinfections in pregnancy relies on the serological testing of blood samples to detect primoinfections. The first test used was SabinFeldman's dye test in the 1970s, but this was soon replaced by the less laborious and cheaper indirect immunofluorescence test (IIFT). Both tests are capable of detecting Toxoplasma-specific antibodies from all immunoglobulin classes (or at least IgG, IgM and IgA).

If test results were negative, in Austria the test was performed once per trimester [3], while France recommended monthly testing throughout pregnancy [10]. Positive results in following tests hinted at primoinfection (seroconversion). If the result was positive for the first test in the first trimester, a sample taken 14 days later was required to check for significant titer rise. If the titer remained stable and if tests for specific IgM were negative, immunity and no future risk was assumed. In the case of significant titer rise and/or detection of specific IgM antibodies, acute infection was considered possible and aid from a reference laboratory was required.

Enzyme-linked immunoassays (EIA) improved mass screening, as these tests can be fully automated to reduce the cost of trained laboratory personnel. From an economic point of view, laboratories now faced the disadvantage that most tests are designed to test the immunoglobulin classes separately. Instead of one "catch-all" IIFT test, for most EIAs at least two tests-for IgG and IgM-were needed to rule out acute infection in seronegative persons. In many countries, the majority of pregnant women are seronegative (e.g. nearly $70 \%$ in Austria [21]). These women need several checks during pregnancy. This requirement may have a decisive impact on the cost of a screening program.

Although IgM-antibodies appear first in acute infections (about 1-2 weeks after infection), production of detectable levels of IgG antibodies will follow soon (within 4 weeks of infection), but some individual variation must be taken into account [22]. Some laboratories decide to test only for Toxoplasma-IgG, unless a positive test leads to additional testing of IgM to rule out acute infection. The diagnostic loss due to the small time frame between the production of IgM and IgG antibodies must be balanced against time loss due to large screening intervals: e.g., the effectiveness of testing IgG monthly may be comparable to testing IgG and IgM every two months. Only particular IgG tests are suitable for laboratories with a first line IgG screening: e.g., IgG antibodies appear earlier in the AxSYM Toxo-IgG tests compared to the Access Toxo-IgG tests in such a way that a ratio taken from both tests helps to distinguish early from late infection [23].

Seroconversion: Routine laboratories can make quite a safe diagnosis of maternal primoinfection in pregnancy even without the aid of a reference laboratory, if the first screening test was clearly negative and a consecutive sample from the same pregnancy yields a clear positive result with high IgG antibody levels (corresponding to IIFT titer levels $>1: 64$ ). In line with this result, the laboratory should expect IgM to be positive and IgG avidity to be low. Afterwards, a significant IgG titer rise supports the diagnosis, if high antibody levels have not been reached at the first seropositive test. If $\operatorname{IgM}$ is positive but $\operatorname{IgG}$ is negative (suggestive of very recent infection), seroconversion to IgG antibodies must be demonstrated in a further sample taken two weeks later. In rare instances, false negative IgM results in some commercially available tests in confirmed seroconversions have been reported [24-26].

Confusion and errors might occur if several laboratories were engaged in screening the same person. Results may not be comparable. The same problem will appear if a laboratory changes its tests (e.g. to test kits from another producer). In addition, results close to borderline zones should be interpreted with care, as seropositive may not be safely distinguished from seronegative, especially with manually read tests like the IIFT. Although EIA tests are not affected by subjective readings, they also give some borderline or close-to-borderline results that may produce conflicting results in serial testing. If economic resources allow the freezing of the first serum sample in pregnancy, retesting this sample with different serological tests may secure the diagnosis of seroconversion.

Single serum testing: In general, it takes several weeks for a woman to become aware of pregnancy and present herself to a doctor to participate in a prenatal care program. If the first sample yields a seropositive result, a negative IgM test rules out infection within the immediately preceding three or four months. If not so, high IgG avidity permits us to draw the same conclusion. Some laboratories secure it by checking a second sample taken two weeks later to ensure that the titer remains stable (i.e., a fourfold titer rise or more would not be concordant with the conclusion) as IgM might turn negative even within three months in a few instances [22] or be false-negative [2426]. Past or preconceptual infection is considered to confer protective immunity throughout childbearing life and no harm to the current pregnancy will be expected.

Confusion may occur due to unspecific signals with the $\operatorname{IgG}$ tests [25], but these are very rare. These women, who are probably susceptible, might be excluded from further screening by erroneously 
assuming immunity. This problem appears to be negligible compared to the much bigger losses in screening programs due to poor compliance.

If the first test takes place late in pregnancy $\left(>16^{\text {th }}\right.$ gestational week), positive $\operatorname{IgG}$ and negative $\operatorname{IgM}$ cannot definitively rule out primoinfections in early pregnancy. Then, some part of the pregnancy remains "blind", i.e., it is not covered by prenatal Toxoplasma screening.

Primoinfection will produce positive IgM followed soon by low $\mathrm{IgG}$ avidity. Unfortunately, this constellation is not indicative of recent primoinfection as positive IgM and low IgG avidity antibodies persist for more than three months up to one year or even more [25-27]: e.g., in a pregnant woman with these results in the 12th gestational week, she will perhaps be as likely to have a past infection as to have an acute infection within her current pregnancy. If the single serum results are IgM positive and IgG negative, this can be due to unspecific IgM signals in the serological test [25]. In this case, this pattern will persist in later serological checks (rechecked after 7-10 days - or even later as some tests detect IgG late in acute infections) and these women should be judged seronegative.

Confirmatory testing: Doubtful results that suggest possible primoinfection require confirmatory testing in reference laboratories. Suspicious single serum results are always challenging, as serological tests must inform retrospectively whether the time point of infection falls into the ongoing pregnancy or not. In addition to $\operatorname{IgM}$ and $\operatorname{IgG}$ avidity (to be performed in most routine laboratories), tests for IgA and IgE, differential agglutination AC/HS [13], AxSYM Toxo-IgG/Access Toxo-IgG ratio [23], and time-dependent reactions in a recombinant line assay [28] help to distinguish pre- and postconception infections. Titer kinetics are particularly difficult to study: "Due to the great individual variation, it seems impossible to estimate when the infection occurred based on results obtained from a single serum, and it may even be difficult to assess when a titer increase in paired sera is detectable unless the first sample is only marginally positive [22]." In addition, titer kinetics might be hindered by specific treatment if a decision for immediate intervention has been taken [25].

Maternal primoinfection is an infrequent incident in pregnancy (e.g. $<0.5 \%$ in Upper Austria [21]) and just for statistical reasons misleading results may be at least as frequent as true infections. Therefore, it is advisable for even experienced laboratories to have all suspicious samples checked in a reference laboratory, even if routine tests were convincing. Nonetheless, in cases of clear seroconversions, a treatment decision should be taken without waiting for confirmation from reference laboratories or further testing of titer kinetics to avoid the possibility that "precious time can be lost in initiating therapeutic treatment to prevent transmission and/or to limit damage to the foetus" [25]. In suspected infections, preliminary treatment should also be considered until confirmatory test results indicate whether to continue or withdraw it.

Reference testing remains cumbersome, especially in single serum testing. In some instances, even reference laboratories will not be able to obtain a conclusive result.

Further management in case of maternal infection: It is beyond the scope of this article to deal with the further medical management of maternal infections in detail. From a public health perspective, it is important to learn that infected pregnant women can be offered antimicrobial treatment to avoid transmission of the pathogen to the fetus and to treat the fetus if already infected. Until the $16^{\text {th }}$ gestational week, only spiramycin can be given. This antimicrobial has the disadvantage that it only prevents transmission. After the $16^{\text {th }}$ [3] or $18^{\text {th }}$ [13] gestational week, folic acid antagonists are another option with a presumed impact on the already infected unborn. Before choosing this treatment, it is possible to explore whether the fetus has already been infected by PCR-testing of amniotic fluid. Unfortunately, due to limited sensitivity, negative PCR results do not rule out congenital infections, especially if treatment with folic acid inhibitors has already been initiated in the mother $[13,27]$.

Assessment of outcomes of maternal and congenital infections is hampered by the fact that sequels due to toxoplasmosis in pregnancy may often not be apparent at birth and may develop late in the course of childhood. Still, more follow-up studies of cases are needed to obtain complete and unbiased information about the outcome of treated and untreated infections [29].

\section{Shortcomings of Current Screening Programs from a Public Health Perspective}

As mentioned before, the effectiveness of early treatment in pregnancy has not been proven by placebo-controlled multicenter studies nor do we know for sure that early treatment really does not work despite several decades of practical experience with screening. Today, public health decision-makers tend to drop Toxoplasma screening in pregnancy due to its allegedly unproven effectiveness despite longlasting and intensive experience and research. To the authors, this attitude appears to be somewhat unfair as public health principles have been neglected in the past, especially an appropriate epidemiological assessment of the programs in Austria and France. Not surprisingly, some shortcomings of Toxoplasma screening can be explained by this negligence:

\section{Poor epidemiological data}

Despite more than three decades of costly population-wide free screening, Austria and France have failed to provide regular (e.g. annual), extensive assessment and reporting on key epidemiological data like seroprevalence, incidence of maternal infections (stratified by gestational week of diagnosis and an estimated time point of infection), incidence of fetal infections (if amniocentesis was performed), treatments, and, most importantly, the clinical outcome in the child in the long run: "The prime example of minimal available data is France ..." [30], but at least France introduced some mandatory reporting in May 2007 [1] and reported some first results about congenital toxoplasmosis [10]. Even worse, Austria is still waiting for these first steps in the right direction. Laboratory reporting of mandatorily reportable diseases could be supported by modern information technology and the incidence of proven maternal infections is not too high to implement an epidemiological follow-up service with individual data. Interestingly, in many European countries toxoplasmosis or congenital toxoplasmosis is a notifiable disease [1] despite the fact that the vast majority of cases will go undetected without widespread screening. The development of some kind of "wild screening" underlines the poor and unconvincing public health concepts in many countries. More reasonable is Switzerland's new approach to at least continue some screening in the Basel region for surveillance purposes [16].

\section{Optimum treatment scheme unclear}

Failure to implement an epidemiological follow-up of proven infections means that there is not only very little systematic information on the outcomes but also about optimum treatment, optimum dosage, optimum duration and probability of side effects. Still, there are different treatment recommendations (e.g., four different treatment schemes 
were used by the participants in the EMSCOT multicenter study [6]), and more published data on treatment-specific outcomes would help to find the most promising scheme.

\section{"Blind periods"}

Recently, we learned that in many regions all the recommended serological checks were completed throughout pregnancy in only 30 $40 \%$ of pregnant women [21,31-33]. When screening is discontinued too early in pregnancy, infections occurring in the remaining time until birth $[25,34]$ will go undetected by the program ("blind periods"). "Blind periods" not only leave the individual fetus without early treatment, but also lead to underreporting and may even introduce bias into studies: Underreporting mainly affects late pregnancies and in the latest European treatment studies, cases from the first and second trimester were overrepresented [6]. The results of these studies cannot be generalized to the third trimester (due to different probabilities of transmission to the fetus and of occurrence of apparent disease $[9,35]$ ). The first epidemiological information from the French surveillance system of congenital toxoplasmosis reports that $59 \%$ of cases were notified as late as "in the neonatal and postnatal periods" [10], i.e., at birth or later, despite seven scheduled free serological checks throughout pregnancy.

\section{Screening intervals are too long}

Although treatment effectiveness was not supported by the SYROCOT study, it did yield some evidence (OR 0.48, $\mathrm{p}=0.05$ ) that treatment started "within three weeks of seroconversion" reduces the rate of materno-fetal transmission of Toxoplasma gondii compared to treatment beginning no earlier than eight weeks later [4]. Recent findings also support this assumption [9]. Some present programs put the main emphasis on early pregnancy and expensive reference testing in doubtful single serum samples; e.g., Austria traditionally recommended only one test per trimester [3], although shorter intervals (maximum eight weeks) were later advocated by Austrian experts $[24,36]$. Changing the emphasis to late pregnancy with short screening intervals while reducing costs per test (e.g. only IgG) could be the more effective approach, as risk of transmission that might be prevented is higher in late pregnancy. In addition, statistical estimation of the time point of infection (to study seasonality [37] and dependency of intrauterine transmission and outcome of disease by gestational week) is greatly improved with short screening intervals. Unfortunately, many investigators do not pay sufficient attention to the fact that time point of diagnosis does not equal time point of infection!

\section{Late start of screening}

The later that screening is initiated in pregnancy, the bigger is the proportional period of pregnancy that goes without the possibility of checking for seroconversion as the most reliable and immediately available marker for maternal primoinfection. If the first sample is taken beyond the $16^{\text {th }}$ gestational week, "blind periods" in early pregnancy will remain for seropositive women with negative IgM and/or high IgG avidity as these tests only rule out acute infection during the immediately preceding three to four months. If confirmatory testing is required (positive IgM and low IgG avidity), the decision becomes harder for a reference laboratory the longer the time span between conception and the first serum sample. Therefore, any screening program must strive for an early first test in pregnancy. For women planning pregnancy (especially those attending in vitro fertilization clinics), serum samples should be examined before conception-if possible three months before. If Toxoplasma primoinfection is suspected, pregnancy should be withheld for the next six months [27].

\section{Adverse effects of false positive alerts}

In practice, there is often a delay of several days or even weeks between suspected primoinfection and definitive diagnosis due to: Mailing of samples to a reference laboratory, some confirmatory tests are not being performed daily in the reference laboratory, control of titer dynamics in second samples are taken about 10-14 days later, and amniocentesis to test for transmission of maternal infection to the fetus. In addition, in unfavorable circumstances, it may be impossible for even a reference laboratory to make a safe, definitive diagnosis. And if maternal infection is proven, a negative PCR from amniocentesis does not rule out infection of the fetus due to limited sensitivity of the test. This may have adverse psychological consequences for the parents due to notable anxiety $[29,38]$. Most problematic are suspected cases without confirmation of prenatal toxoplasmosis in the long run, because they are affected by the adverse effects of the screening without having any potential benefit. The more intensively screening is performed the more often unspecific positive test results will produce false alerts. In addition, unnecessary amniocentesis or induced termination of pregnancy may result from erroneously suspected infections [38].

\section{Discussion and Recommendations}

Today, Toxoplasma screening in pregnancy stands at a crossroads in several countries, e.g., Austria is waiting for a decision from its Ministry of Health about whether to discontinue the eldest program that is free for the entire population (personal communication: Prof. Dr. Herbert Auer, Institute of Specific Prophylaxis and Tropical Medicine at the Medical University of Vienna). In the authors' opinion, poor performance was due to, firstly, the neglect of public health principles and, secondly, inappropriate consideration of specific limitations of laboratory tests:

Firstly: Public health authorities should have provided feasible, quality-controlled structures and processes that promise optimum results (Table 1) e.g., Austria should have installed an epidemiological unit to analyze all necessary epidemiological clue markers, as mentioned above. On behalf of the Ministry of Health, the Austrian Agency of Health and Food Safety (AGES) is commissioned and dedicated to the epidemiology of many zoonotic diseases in general, but interestingly not to toxoplasmosis in pregnancy. Another structural defect is the ambiguous nomination of a central reference laboratory: For many years, two laboratories at the Medical University of Vienna have competed for this function (the Toxoplasma Laboratory at the Department of Neonatology and Pediatric Intensive Care at University Children's Hospital and the Department of Medical Parasitology at the Institute of Specific Prophylaxis and Tropical Medicine). A third structural defect has been poor support to encourage data flow by mandatory reporting. Without legal measures, the strict Austrian personal data protection legislation hinders the forwarding of personal data from the electronic databases of routine laboratories to a central epidemiological unit. To provide optimum processes, close collaboration with routine laboratories that participate in the screening program is necessary. Electronic reminder systems and inclusion in the program of a final screening test at birth clinics should get around the problem of "blind periods". In addition, efforts should be made to have the first screening test as soon as possible in pregnancy. The efficiency of avoiding "blind periods" should be controlled by analysis of anonymized data sets by the central epidemiological unit. Delays in forwarding blood samples from the attending physician to the routine laboratory and, in cases of suspected infection, from the routine laboratory to the reference laboratory must be kept to a minimum. 


\begin{tabular}{|c|c|}
\hline Recommendation & Further comments \\
\hline $\begin{array}{l}\text { Implement a central } \\
\text { epidemiological unit }\end{array}$ & $\begin{array}{l}\text { - To inform about seroprevalence and maternal } \\
\text { infections } \\
\text { - To inform about prenatal infections in offspring } \\
\text { - To inform about treatment and long-term } \\
\text { outcomes of prenatal infection } \\
\text { - To control compliance with the screening } \\
\text { schedule }\end{array}$ \\
\hline $\begin{array}{l}\text { Introduce mandatory } \\
\text { reporting of all relevant data } \\
\text { to the epidemiological unit }\end{array}$ & $\begin{array}{l}\text { or require informed consent from pregnant } \\
\text { women who want to participate in the free } \\
\text { prenatal care program to prevent obstacles from } \\
\text { personal data protection legislation }\end{array}$ \\
\hline $\begin{array}{l}\text { Implement a single } \\
\text { reference laboratory }\end{array}$ & \\
\hline $\begin{array}{l}\text { Nominate screening } \\
\text { laboratories }\end{array}$ & 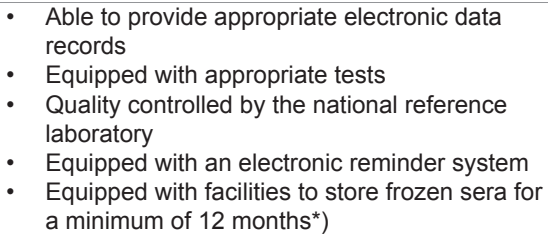 \\
\hline \multicolumn{2}{|l|}{$\begin{array}{l}\text { Include birth clinics (test at } \\
\text { delivery!) in the screening } \\
\text { program }\end{array}$} \\
\hline $\begin{array}{l}\text { Maintain close contact } \\
\text { with prenatal care doctors } \\
\text { and their representatives } \\
\text { to foster adherence to the } \\
\text { national screening scheme }\end{array}$ & \\
\hline $\begin{array}{l}\text { Decide on shortest possible, } \\
\text { feasible, and affordable } \\
\text { screening intervals }\end{array}$ & \\
\hline
\end{tabular}

*) also helpful to confirm prenatal infections from other pathogens like rubella virus, cytomegalovirus, or Treponema pallidum.

Table 1: Recommendations for Public Health Decision Makers.

Secondly: Single serum testing is demanding (difficult diagnosis, expensive special test, time consuming). There should be more emphasis on detecting seroconversions, especially in the second and third trimesters, as often a sufficiently safe diagnosis can be made immediately to decide about early treatment to prevent transmission to the fetus. Later confirmatory testing from a reference laboratory will just be a matter of care and quality control and should not delay urgent intervention. Therefore, routine laboratories should be able to test for IgM, IgG avidity, and have two different tests for IgG (to confirm seroconversion by a second test). Frozen sera from first samples in pregnancy may support confirmatory testing of seroconversions. Shorter screening intervals with IgG tests may have more impact than less IgG/IgM testing. Development of point-of-care tests from capillary blood could improve compliance and the feasibility of short-interval screening.

The crucial question about treatment effectiveness must be studied further, and the countries with a screening tradition, i.e., Austria and France, should assume responsibility for world-wide toxoplasmosis research, contributing more and better-defined study cases by improving their screening programs. Appropriate controls for Austrian cases might be found in birth clinics in the German province of Bavaria as its population is culturally, socially, and genetically quite similar to the Austrian people. Mother-child care documents of all enrolled children could be examined at the age of three years for signs of neurological disorders or eye disease. If these are found, detailed examinations for probable congenital toxoplasmosis should follow. If toxoplasmosis screening in pregnancy is effective, the incidence of disease should be lower in Austrian than in Bavarian children.
The fact that many European countries without a clear national mandate have the phenomenon of "wild screening" underlines the unwillingness of experts and physicians to accept the reluctant or disinterested attitude of public health officials owing to inconclusive study results.

In conclusion, close collaboration and mutual understanding between public health experts, prenatal care and birth clinic physicians, and medical microbiologists can overcome decisive past shortcomings in screening for Toxoplasma primoinfections in pregnancy.

\section{Acknowledgment}

We are indebted to Prof. Dr. Herbert Auer, Institute of Specific Prophylaxis and Tropical Medicine at the Medical University of Vienna for critical reading of the manuscript.

\section{References}

1. Bénard A, Petersen E, Salamon R, Chêne G, Gilbert R, et al. (2008) Survey of European programmes for the epidemiological surveillance of congenital toxoplasmosis. Eurosurveillance 13

2. Leroy V, Raeber PA, Petersen E, Salmi LR, Kaminski M, et al. (2005) National public health policies and routine programs to prevent congenital Toxoplasmosis, Europe, 2005. Unpublished report, Bordeaux, France.

3. Aspöck H, Pollak A (1992) Prevention of Prenatal Toxoplasmosis by Serological Screening of Pregnant Women in Austria. Scand J Infect Dis Suppl 84: 32-37.

4. Thiébaut R, Leproust S, Chêne G, Gilbert R, SYROCOT (Systematic Review on Congenital Toxoplasmosis Study Group) (2007) Effectiveness of prenatal treatment for congenital toxoplasmosis: a meta-analysis of individual patients data. Lancet 369: 115-122.

5. Gras L, Wallon M, Pollak A, Cortina-Borja M, Evengard B, et al. (2005) Association between prenatal treatment and clinical manifestations of congenital toxoplasmosis in infancy: a cohort study in 13 European centres. Acta Paediatr 94: 1721-1731.

6. Gilbert R, Gras L, European Multicentre Study on Congenital Toxoplasmosis (2003) Effect of timing and type of treatment on the risk of mother to child transmission of Toxoplasma gondii. BJOG 110: 112-120.

7. Gilbert R, Gras L, Wallon M, Peyron F, Ades AE, et al. (2001) Effect of prenatal treatment on mother to child transmission of Toxoplasma gondii: retrospective cohort study of 554 mother-child pairs in Lyon, France. Int J Epidemiol 30 1303-1308.

8. Thiébaut R, Leroy V, Alioum A, Binquet C, Poizat G, et al. (2006) Biases in observational studies of the effect of prenatal treatment for congenital toxoplasmosis. Eur J Obstet Gynecol Reprod Biol 124: 3-9.

9. Hotop A, Hlobil H, Gross U (2012) Efficacy of rapid treatment initiation following primary Toxoplasma gondii infection during pregnancy. Clin Infect Dis 54: 15451552

10. Villena I, Ancelle T, Delmas C, Garcia P, Brézin AP, et al. (2010) Congenital toxoplasmosis in France in 2007: First results from a national surveillance system. Euro Surveill 15.

11. Havelaar AH, Kemmeren JM, Kortbeek LM (2007) Disease burden of congenital toxoplasmosis. Clin Infect Dis 44: 1467-1474.

12. Montoya JG, Liesenfeld O (2004) Toxoplasmosis. Clin Infect Dis 47: 554-566.

13. Montoya JG, Remington JS (2008) Management of Toxoplasma gondii Infection during Pregnancy. Clin Infect Dis 47: 554-566.

14. Remington JS, McLeod R, Thulliez P, Desmonts G (2006) Toxoplasmosis. In Infectious Diseases of the Fetus and the Newborn Infant. (6thedn), Elsevier Saunders, Philadelphia.

15. Schmidt DR, Hogh B, Andersen O, Fuchs J, Fledelius H, et al. (2006) The national neonatal screening programme for congenital toxoplasmosis in Denmark: results from the initial four years, 1999-2003. Arch Dis Child 91: 661665.

16. Rudin C, Boubaker K, Raeber PA, Vaudaux B, Bucher HC, et al. (2008) Toxoplasmosis during pregnancy and infancy. A new approach for Switzerland. Swiss Med Wkly 138 1-8.

17. Kieffer F, Wallon M, Garcia P, Thulliez P, Peyron F, et al. (2008) Risk factors fo 
Citation: Sagel U, Krämer A (2013) Screening of Maternal Toxoplasmosis in Pregnancy: Laboratory Diagnostics from the Perspective of Public Health Requirements. J Bacteriol Parasitol S5-003. doi:10.4172/2155-9597.S5-003

Page 6 of 6

retinochoroiditis during the first 2 years of life in infants with treated congenital toxoplasmosis. Pediatr Infect Dis J 27: 27-32.

18. McLeod R, Kieffer F, Sautter M, Hosten T, Pelloux H (2009) Why prevent, diagnose and treat congenital toxoplasmosis? Mem Inst Oswaldo Cruz 104: 320-344.

19. Cortina-Borja M, Tan HK, Wallon M, Paul M, Prusa A, et al. (2010) Prenatal treatment for serious neurological sequelae of congenital toxoplasmosis: An observational prospective cohort study. PLoS Med 7: e1000351.

20. Gilbert R (2009) Treatment for congenital toxoplasmosis: finding out what works. Mem Inst Oswaldo Cruz 104: 305-311.

21. Sagel U, Krämer A, Mikolajczyk RT (2011) Incidence of maternal Toxoplasma infections in pregnancy in Upper Austria, 2000-2007. BMC Infect Dis 11: 348.

22. Jenum PA, Stray-Petersen B (1998) Development of specific immunoglobulins $\mathrm{G}, \mathrm{M}$, and A following primary Toxoplasma gondii infection in pregnant women. J Clin Microbiol 36: 2907-2913.

23. Flori P, Bellete B, Crampe C, Maudry A, Patural H, et al. (2008) A technique for dating toxoplasmosis in pregnancy and comparison with the Vidas antiToxoplasma IgG avidity test. Clin Microbiol Infect 14: 242-249.

24. Auer H, Vander-Möse A, Aspöck H (2003) The confusing diversity of IgM tests in the diagnosis of Toxoplasma infections: Efforts towards an optimal strategy. Wien Klin Wochenschr 115: 18-22.

25. Sensini A (2006) Toxoplasma gondii infection in pregnancy: Opportunities and pitfalls of serological diagnosis. Clin Microbiol Infect 12: 504-512.

26. Roberts A, Hedman K, Luyasu V, Zufferey J, Bessières MH, et al. (2001) Multicenter evaluation of strategies for serodiagnosis of primary infection with Toxoplasma gondii. Eur J Clin Microbiol Infect Dis 20: 467-474.

27. Reiter-Owona I (2005) Laboratory diagnosis of toxoplasmosis-possibilities and limitations. J Lab Med 29: 439-445.

28. Pfrepper KI, Enders G, Gohl M, Krczal D, Hlobil H, et al. (2005) Seroreactivity to and avidity for recombinant antigens in toxoplasmosis. Clin Diagn Lab Immuno 12: $977-982$

29. Freeman K, Salt A, Prusa A, Malm G, Ferret N, et al. (2005) Association between congenital toxoplasmosis and parent-reported developmental outcomes, concerns, and impairments, in 3 year old children. BMC Pediatrics 5: 23.

30. Pappas G, Roussos N, Falagas ME (2009) Toxoplasmosis snapshots : Global status of Toxoplasma gondii seroprevalance and implications for pregnancy and congenital toxoplasmosis. Int J Parasitol 39: 1385-1394.

31. Sagel U, Krämer A, Mikolajczyk RT (2012) "Blind periods" in screening for toxoplasmosis in pregnancy in Austria-a debate. BMC Infect Dis 12: 118

32. Cornu C, Bissery A, Malbos C, Garwig R, Cocherel C, et al. (2009) Factors affecting the adherence to an antenatal screening programme: An experience with toxoplasmosis screening in France. Euro Surveill 14

33. De Paschale M, Agrappi C, Manco MT, Cerulli T, Clerici P (2010) Implementation of screening for Toxoplasma gondii in pregnancy. J Clin Med Research 2: 112 116.

34. Lago EG, de Carvalho RL, Jungblut R, da Silva VB, Fiori RM (2009) Screening for Toxoplasma gondii antibodies in 2,513 consecutive parturient women and evaluation of newborn infants at risk for congenital toxoplasmosis. SciMed 19 27-34.

35. Dunn D, Wallon M, Peyron F, Petersen E, Peckham C et al (1999) Motherto-child transmission of toxoplasmosis : Risk estimates for clinical counselling Lancet 353: 1829-1833.

36. Prusa AR, Hayde M, Gerstl N, Pollak A (2005) Infection with Toxoplasma gondi during pregnancy. Gynäkologische Praxis 29: 41-44.

37. Sagel U, Krämer A, Mikolajczyk RT (2010) Seasonal trends in acute toxoplasmosis in pregnancy in the federal state of Upper Austria. Clin Microbio Infect 16: 515-517.

38. Khoshnood B, De Vigan C, Goffinet F, Leroy V (2007) Prenatal screening and diagnosis of congenital toxoplasmosis: A review of safety issues and psychological consequences for women who undergo screening. Prenat Diagn 27: $395-403$
This article was originally published in a special issue, Diagnostic Methods of Microbial Pathogens handled by Editor(s). Stefan Borgmann, Hospital of Ingolstadt, Germany 